\title{
Dosimetric Comparison of Passive Scattering and Active Scanning Proton Therapy Techniques Using GATE Simulation
}

\section{Ali Asadi}

Sharif University of Technology

\section{Azadeh Akhavanallaf}

Geneve University Hospitals: Hopitaux Universitaires Geneve

Seyed Abolfazl Hosseini ( $\nabla$ sahosseini@sharif.edu )

Sharif University of Technology

Naser Vosoughi

Sharif University of Technology

\section{Habib Zaidi}

Geneva University Hospitals: Hopitaux Universitaires Geneve

\section{Original research}

Keywords: Proton therapy, Dosimetry, PSPT, PBS, GATE, TG-119.

Posted Date: September 2nd, 2021

DOl: https://doi.org/10.21203/rs.3.rs-847332/v1

License: (c) (1) This work is licensed under a Creative Commons Attribution 4.0 International License. Read Full License 


\section{Abstract}

Background: In this study, two proton beam delivery designs, i.e. passive scattering proton therapy (PSPT) and pencil beam scanning (PBS), were quantitatively compared in terms of dosimetric indices. The GATE Monte Carlo (MC) particle transport code was used to simulate the proton beam system; and the developed simulation engines were benchmarked with respect to the experimental measurements.

Method: A water phantom was used to simulate system energy parameters using a set of depth-dose data in the energy range of 120-235 MeV. To compare the performance of PSPT against PBS, multiple dosimetric parameters including Bragg peak width $\left(\mathrm{BP}_{\mathrm{W} 50}\right)$, peak position, range, peak-to-entrance dose ratio, and dose volume histogram have been analyzed under the same conditions. Furthermore, the clinical test cases introduced by AAPM TG-119 were simulated in both beam delivery modes to compare the relevant clinical values obtained from Dose Volume Histogram (DVH) analysis.

Results: The parametric comparison in the water phantom between the two techniques revealed that the value of peak-to-entrance dose ratio in PSPT is considerably higher than that from PBS by a factor of $8 \%$. In addition, the $\mathrm{BP}_{\mathrm{W} 50}$ in PSPT was increased by a factor of 7\% compared to the corresponding value obtained from PBS model. TG-119 phantom simulations showed that the difference of PTV mean dose between PBS and PSPT techniques are up to $1.8 \%$ while the difference of max dose to organ at risks (OARs) exceeds $50 \%$.

Conclusion: The results demonstrated that the active scanning proton therapy systems was superior in adapting to the target volume, better dose painting, and lower out-of-field dose compared to passive scattering design.

\section{Introduction}

Proton therapy (PT) due to their excellent dose distribution can significantly reduce the absorbed dose by the patient's body relative to the photon beams (1). PT was first initiated in 1954 and is currently going as part of modern radiation therapy technologies in many developed countries (2). In PT, there are two main techniques of irradiation, namely active scanning or Pencil Beam Scanning (PBS) and Passive Scattering Proton Therapy (PSPT). Passive scattering facilities are composed of specific mechanical devices in the particle trajectory to shape the beam to the tumor volume using particle-matter interactions. The scattering interaction spread the originally Gaussian-distributed beam to shape a wide homogeneous beam to the tumor using patient-specific collimators. A rotating wheel of varying thickness, called range modulator, is used to generate a uniform Spread-Out Bragg Peak (SOBP). Lastly, proton beam travel through a longitudinal compensator, a specific scatterer device, specifically drilled for each field and each patient, to achieve the last conformal shaping of the beam just before the skin of the patient. Nowadays, proton beam delivery is switching from the use of passively scattered mode to pencil beam scanning owing to the feasibility of more conformal dose to tumors and higher dose rate in PBS compared to PSPT (3). In addition, pencil beam technique can reduce the need for specific mechanical hardware required in 
PSPT. Another advantage of the active scanning technique is related to the lower level of secondary particles, mostly neutrons that are generated from the interaction of primary protons with multiple scattering components. In PBS, multiple magnets in $\mathrm{x}$ and $\mathrm{y}$ directions, based on the charge of particles, are used to drift the beam and scanning the target volume spot by spot with a 3D narrow pencil beam (4, 5). There are some studies in biological dose comparison between active and passive scanning proton therapy techniques in the cellular level $(3,6,7)$. Gridley et al. (7) compared cell response to active scanning and passive beam delivery techniques. They reported lower dose values at the entrance region, a sharper fall-off and a higher dose rate for active scanning mode. While they did not find any considerable difference in relative biological effectiveness (RBE) between two methods. Michaelidesova et al. (3) reported that between two techniques, there is neither considerable beam quality difference from MC simulation, nor statistically significant difference in biological endpoints. Nomura et al. (6) reported a higher Linear Energy Transfer (LET) at distal region in active scanning mode compared to passive scattering method. To the best of our knowledge, the current study is the first report that systematically compares physical doses between two modes of proton therapy. In this work, multiple dosimetric criteria such as $\mathrm{BP}_{\mathrm{W} 50}$, peak position, range, peak-to-entrance dose ratio, etc. have been analyzed. Furthermore, clinically relevant dose parameters have been compared through TG-119 clinical test cases.

\section{Material And Method}

\section{Beam modeling}

In this work, two main proton therapy techniques, i.e. PBS and PSPT, have been simulated using Monte Carlo GATE code. Shanghai Advanced Proton Therapy (SAPT) facility, a synchrotron-based active scanning proton therapy system, was simulated. Figure 1. Depicts the geometrical characteristics of SIEMENS IONTRIS system at SAPT. In this system proton beams are extracted from synchrotron and drifted to the nozzle, by using the paired scanning magnets in horizontal $(X)$ and vertical $(Y)$ directions. Proton beam spot is moved around the isocenter with energies between 70-235 MeV (8). The advantage of this technique against scattering-based technique is that a range shifter is not required to shape the beam to the tumor volume, because the synchrotron accelerate the protons slowly and conform the tumor dose in the dimension lateral to the beam (8). The system characteristics are listed in Table 1. Dose delivered to the phantom is monitored in real time by using two parallel-plate ionization chambers. Spot size and beam optic are measured by using the position detectors. Unlike discrete scan mode (pixel scan), IONTRIS provided a continuous beam scan mode (raster scan). 
Table 1

The characteristics of SAPT proton therapy system obtained from (8).

\begin{tabular}{|ll|}
\hline Item & value \\
\hline Energy $(\mathrm{MeV})$ & $70.0-235.0$ \\
\hline Field size $\left(\mathrm{cm}^{2}\right)$ & $30.0 \times 40.0$ \\
\hline Scanning magnet $x$ to isocenter distance $(\mathrm{cm})$ & 287.0 \\
\hline Scanning magnet $y$ to isocenter distance $(\mathrm{cm})$ & 242.0 \\
\hline Nozzle to isocenter distance $(\mathrm{cm})$ & 40.0 \\
\hline Scan speed in $\mathrm{x}(\mathrm{cm} / \mathrm{ms})$ & 2.0 \\
\hline Scan speed in $\mathrm{y}(\mathrm{cm} / \mathrm{ms})$ & 0.5 \\
\hline Dose rate $(\mathrm{Gy} / \mathrm{min})$ & 2.0 \\
\hline
\end{tabular}

Passive scattering system geometry was obtained from (9). In this design, proton beam passes through the vacuum window, first scatterer, first monitor, range modulator wheel, second scatterer, second monitor, collimator, and the size-changeable snout (brass tube). The first scatterer is set for spreading the beam laterally and the range modulator wheel spread the beam longitudinally. Two jaws can provide a sizechangeable treatment field, while the aperture can control the lateral conformity of the beam.

The strategy reported by Grevillot et al. (10) has been adopted for PBS system simulation. a complete treatment plan in PENCIL BEAM GATE SOURCE TPS including two main components, source description and plan description has been defined $(10,11)$. In PBS simulation, the source description file defines the beam delivery system with a set of the polynomial equations which allows calculation of the optical and energy properties of each pencil beam at the nozzle output as a function of beam energy. It consists of eight equations; where, two equations describe energy properties and the rest of the equations describe optical properties. The plan description file contains one or more fields described by gantry angle and a set of pencil beams.

In PSPT design, the simulated geometry consists of a nozzle and a water phantom (Fig. 2) (9). The system characteristics including the nozzle components and materials, are listed in Table 2. All the components are simulated in mm accuracy. For the proton beam, the mean primary energy was set on 212.2 MeV, with a Gaussian distribution of 3.5\%. In the simulation, proton beam, before entering to the nozzle, passes from $10 \mathrm{~cm}$ air, and after the exit window, passes from $57 \mathrm{~mm}$ air, just before entering to the water phantom. 
Table 2

Specifications of simulated PSPT nozzle.

\begin{tabular}{|lllll|}
\hline Component & Material & $\begin{array}{l}\text { Length } \\
(\mathbf{m m})\end{array}$ & Outer radius (mm) & Inner radius (mm) \\
\hline Range shifter tube & Brass & 254 & 82.6 & 76.2 \\
\hline First collimator & Brass & 10 & 76.2 & 6.4 \\
\hline Range shifter & Lexan & A & 62.5 & - \\
\hline Second collimator & Brass & 10 & 57.2 & 12.7 \\
\hline Monitor chamber tube & Brass & 681 & 57.2 & 51.4 \\
\hline Vacuum window & Aluminum & 0.25 & 254 & - \\
\hline Taper tube & Brass & 203 & 44.3 & 38.5 \\
\hline Circular aperture & Brass & 161 & 44.3 & b \\
\hline Half-Circular aperture & Brass & 9.5 & 20 & 12.5 \\
\hline Monitor chamber plate & Brass & 9.5 & 20 & \\
\hline
\end{tabular}

a: is the range shifter length e and varies in the simulation.

b: Internal and external dimensions vary depending on the therapeutic.

Brass $\left(8.49 \mathrm{~g} / \mathrm{cm}^{3}\right)$ is composed of $61.5 \%$ Copper, $35.2 \%$ Zinc and $3.3 \%$ lead; Lexan density:

$1.2 \mathrm{gr} / \mathrm{cm}^{3}$

In this study, two independent Monte Carlo-based simulators for PBS and PSPT have been developed and benchmarked against experimental measurements reported in the literature. GATE code, a multipurpose Monte Carlo code based on the libraries of the Geant4 toolkit, has been used in this simulation (version 8.2 and QGSP-BERT-EMZ physical list) (12)). The physical list selected for proton transport simulation is QGSP-BERT-EMZ.

\section{Clinical phantom study}

The clinical test suite recommended by TG-119 includes structures for prostate, head and neck (H\&N), and C-shape cases. The prostate phantom, uses the CTV, PTV, rectum, and bladder; the head \& neck case, includes the PTV, cord, and parotide (left and right); and the C-shape phantom uses a PTV, and a core structure. The PTV in C-shape phantom wrapped around a core, whose outer surface is $5 \mathrm{~mm}$ from the inner surface of the PTV (13).

\section{Plan description}

For a given radiation field, there are two main objectives: maximizing the uniform dose at the target and minimizing the non-target dose (14). Inverse planning algorithm proposed by Sánchez et al. (15) applied for spot and beam selection. The objective function and target in this optimization was set according to 
the TG-119 report (16) and other references $(15,17,18)$. The accuracy of the dose volume histogram obtained from GATE simulation was benchmarked against the results reported by Sánchez et al (15). Hence, clinically relevant dosimetric parameters were compared in terms of mean relative error using Eq. (1):

$$
\operatorname{MRE}=\left(\frac{D_{i}-D_{i}{ }^{\prime}}{D_{i}}\right) \times 100
$$

where, $D_{i}$ represents the dose parameters from our simulation and $D_{i}^{\prime}$ represents the reference data.

For the TG-119 C-shape phantom the dose goals was set base on the PTV ( D95 = 50 Gy, D10 < 55 Gy), and for core (D10<10 Gy). A single proton field was set on the target to maximize the biological effect (15). For H\&N case 70 GyRBE dose (in 35 fraction) was prescribed to be delivered to the PTV70 $\left(D_{20}<55\right.$ GyRBE, $D_{99}<46.5$ GyRBE, and $D_{90}=50$ GyRBE). While maximum dose goal to the OARs were restricted as following: dose to the cord (max $<50$ GyRBE), brain stem ( $\max <54$ GyRBE) and for parotide $\left(D_{50}<20\right.$ GyRBE). To minimize the biological effect on non-target volumes, two filed with 50, and 310 degree angles were set $(15,17)$. In the prostate case, prescribe dose was selected 78 GyRBE (in 39 fraction) (15, 18 ), with the goal dose to PTV ( $D_{5}<83 G y$, and $D_{95}>75.6 \mathrm{~Gy}$ ). Dose to rectum was set to $V 70_{\text {GyRBE }}<30 \%$ and $\mathrm{V} 50_{\mathrm{GyRBE}}<60 \%$. Dose to bladder was restricted by $\mathrm{V} 70_{\mathrm{GyRB}}<35 \%, \mathrm{~V} 50_{\mathrm{GyRB}}<60 \%$, and for femur it was defined $V 50_{\text {GyRBE }}<5 \%$. Two parallel opposed field was used to minimize the dose effect on the normal tissue. During the planning, relative biological effect of protons was applied by a constant factor of 1.1 .

\section{Quantitative analysis}

Both PSPT and PBS facilities were benchmarked against experimental data in terms of depth dose curve and SOBP plan. The average point-to-point difference and statistical analysis (T-test) between each series of data were calculated. When the MC simulators of two systems have been validated, they were compared under the same simulation parameters using water phantom and TG-119 test cases. The physical dose parameters including $\mathrm{BP}_{\mathrm{W} 50}$, peak location, range, and peak-to-entrance dose ratio were compared. In addition, based on the SOBP plan, the factor of

$$
\omega=\sum_{i=1}^{N}\left(\frac{|d i-d r e f i|}{\text { drefi }} \times \frac{\Delta}{L}\right)
$$

Where $d i$ and drefi refers to the simulation and measurement, $\Delta$ is the step between two point, and $L$ is the maximum range.

conformity and penumbra were compared. Furthermore, DVH-driven clinical parameters obtained from TG-119 simulation were compared between two proton therapy techniques.

\section{Results And Discussion}




\section{Experimental study}

Figure 3 shows a comparison between the simulated Integrated Depth Dose (IDD) at the energy of 161.1 $\mathrm{MeV}$ for PBS system, and 212.2 MeV for PSPT system, and the experimental data obtained from $(8,9)$. The simulation results illustrate a good agreement with the experimental measurements. For the PBS system, the mean point-to-point difference between the simulation and measurement was $1.5 \%$ and no statistically significant difference was observed ( $P$-value $=0.008$ ), while for PSPT system the mean pointto-point difference was calculated about $2.76 \%(P-v a l u e=0.048)$.

Fig 4. Demonstrates a good agreement between the simulated SOBP and experimental data for both PBS and PSPT technique. The mean relative error for PBS was calculate about $0.97 \%$ ( $P$-value $=0.003$ ) while for PSPT was $1.08 \%(P-$ value $=0.05)$.

Fig. 5 compares the simulated Bragg peak location, range (R80), $\mathrm{BP}_{\mathrm{W} 50}$ and peak-to-entrance dose ratio between PBS and PSPT beam delivery methods in different energies under the same conditions. Braggpeak location between the two methods had a mean relative difference of $0.74 \%$ with a maximum difference of $0.99 \%$ (P-value $=0.0021$ ). Accordingly, no significant difference on the energy discharge location has been observed between two methods. The parameter of proton beam range in the scattering is slightly longer than the active scanning method with an average difference of $1.8 \mathrm{~mm}$ (maximum difference $2.9 \mathrm{~mm}$ ). In addition, comparison of $\mathrm{BP}_{\mathrm{W} 50}$ parameter between scattering system versus active scanning technique show a considerable difference of about $8 \%$ (P-value $=0.0022$ ). The ratio of peak to entrance dose (normalized) is considerably higher with an average difference of $6.45 \%$ (ranging between $1.9-11.11 \%$ ) in PSPT compared to PBS (P-value=0.0258).

SOBP plan and depth-dose curve in water phantom under the same conditions are compared between PBS and PSPT in Fig. 6 . The mean relative difference in IDD profile between two methods was calculated about 9.3\% (P-value $=0.0001$ ). In PSPT mode, the slope of the fall-down in SOBP curve (normalized) is -0.03 and in PBS mode the slope of the fall-down is -0.08 . The physical range extracted from SOPB curve in the passive scattering method $(354.73 \mathrm{~mm})$ compared to PBS mode $(353.37 \mathrm{~mm})$ is about $1.36 \mathrm{~mm}$ larger. In addition, the value of discharged dose in the modulation region for PBS mode is about $4 \%$ higher than that calculated in PSPT mode. The entrance dose in PBS mode (0.549) was lower by a factor of $13.4 \%$ compared to simulated passive scattering design (0.623).

\section{Clinical study}

Dose distribution for TG-119 phantoms in the pencil beam scanning proton therapy compared to passive scattering model along with DVH analysis for targets and OARs are illustrated in Figs. 7 and 8 . The clinically relevant dose parameters between two modes of proton therapy have been compared in Table 3. The results showed that the difference of PTV mean dose between PBS and PSPT techniques are up to $1.8 \%$ while the difference of max dose to organ at risks (OARs) exceeds $50 \%$ for the spinal cord in $\mathrm{H} \& \mathrm{~N}$ case-study. 
Table 3

dose parameter obtained from dose volume histogram data

\begin{tabular}{|c|c|c|c|c|c|}
\hline Phantom & Organ & Indices & PSPT & PBS & Dif (\%) \\
\hline \multirow[t]{9}{*}{ C-shape } & \multirow[t]{6}{*}{ PTV } & Mean dose (Gy) & 50.4 & 49.5 & -1.8 \\
\hline & & max dose (Gy) & 53.7 & 53.1 & -1.1 \\
\hline & & D 98\% (Gy) & 49.2 & 48.9 & -0.61 \\
\hline & & D 95\% (Gy) & 49.5 & 50.1 & 1.2 \\
\hline & & D 50\% (Gy) & 51.45 & 51.45 & 0 \\
\hline & & D 2 \% (Gy) & 52.1 & 52.2 & 0.2 \\
\hline & \multirow[t]{3}{*}{ Core } & Mean dose (Gy) & 5.21 & 4.177 & -1.03 \\
\hline & & Max dose (Gy) & 33.06 & 19.751 & -13.3 \\
\hline & & Min dose (Gy) & 0 & 0 & 0 \\
\hline \multirow[t]{12}{*}{ Prostate } & \multirow[t]{4}{*}{ Bladder } & V80 & 0 & 0 & 0 \\
\hline & & V75 & 7.161 & 2.6 & -4.56 \\
\hline & & V70 & 9.33 & 3.6 & -159.1 \\
\hline & & V65 & 9.71 & 4.5 & -115.7 \\
\hline & \multirow[t]{4}{*}{ Rectum } & V75 & 14.92 & 6.28 & -137.5 \\
\hline & & V70 & 16.75 & 8.50 & -97 \\
\hline & & V65 & 17.63 & 8.73 & -101.9 \\
\hline & & V60 & 18.28 & 9.01 & -102.9 \\
\hline & \multirow[t]{3}{*}{ PTV } & D95 (Gy) & 76.7 & 75.44 & -1.26 \\
\hline & & D5(Gy) & 81.07 & 78.1 & -3.8 \\
\hline & & Mean dose (Gy) & 79 & 77.8 & -1.2 \\
\hline & Cord & Max & 13.5 & 9 & -50 \\
\hline \multirow[t]{6}{*}{$\mathrm{H} \& \mathrm{~N}$} & L parotide & V30 & 69.5 & 49 & -41.8 \\
\hline & & V40 & 41.22 & 28 & -47.2 \\
\hline & \multirow[t]{2}{*}{$\mathrm{R}$ parotide } & V30 & 84 & 53 & -58.4 \\
\hline & & V40 & 65 & 29 & -124.1 \\
\hline & \multirow[t]{2}{*}{ PTV } & D95 (Gy) & 68 & 67.6 & -0.6 \\
\hline & & D5(Gy) & 70 & 70.2 & 0.28 \\
\hline
\end{tabular}




\begin{tabular}{|llllll|}
\hline Phantom & Organ & Indices & PSPT & PBS & Dif (\%) \\
& & Mean dose (Gy) & 70.2 & 69.6 & -0.85 \\
\hline
\end{tabular}

\section{Discussion And Conclusion}

Nowadays, the treatment of cancerous tumors by particles is increasing due to their outstanding features in the delivery of tailors. In this study, the main methods of beam delivery in proton therapy were simulated, and essential quantities such as peak Bragg location, range, $\mathrm{BP}_{\mathrm{W} 50}$, the ratio of dose at peak to input, and spread out Bragg peak on the target volume in a cubic water phantom were evaluated at different energies. Furthermore, the DVH analysis based on the clinical TG-119 test cases have been investigated. According to our results, some advantages in terms of physical dose parameters in active scanning model compared to passive scattering mode has been observed. However, factor of proton range and peak location were not significantly different between the two nozzle designs. A sharper slope of fall-down in SOBP curve was observed in PBS mode compared to PSPT enabling a more controllable performance to adapt dose to the target volume; where, the sharper the curve, the higher the beam control and the better the dose adjustment on the target area. $\mathrm{BP}_{\mathrm{W} 50}$ in depth-dose profile was on average $8 \%$ larger in scattering system compared to active scanning mode which stems from scattering events and secondary particle generation in the trajectory of proton beam. The ratio of peak-to-entrance dose within the target volume was another investigated quantity in the present work. By comparing this quantity, we found that the inactive dispersion of the transmitters deposited in the skin and surrounding healthy tissue was significantly lower in the active scanning method. The total deposited energy in the out-ofmodulation region in PSPT was about $20 \%$ higher than PBS mode (Fig. 6, area under SOBP curve); which is an important advantage of PBS in clinical application. It can be seen that in the active scanning method, the tumor volume can be better covered and less stitches can be delivered to healthy tissues. Based on the DVH-driven parameters (Table 3), it can be seen that the capability of the PBS mode to reduce the dose to non-target volumes is superior to PSPT while the total dose to body contour is reduced in average about $29.9 \%$ in three TG-119 test cases. In the high dose region of bladder in prostate DVH that is quantified by V75, V70 and V65 indices, the outperformance of PBS versus PSPT has been illustrated. In addition, better conformity of dose to PTV is observed from the fall-down slope in DVH analysis (Fig. 8).

In the present study, the results of the simulation of pencil beam and scattering systems were validated against the experimental data. Therefore, we believe that the simulations performed in this study have the capability to be utilized as independent dose engine simulator of the given systems and applicable in the quality assurance process. Furthermore, under the same conditions and in a homogeneous water phantom, the quantities related to both dose and adaptability properties were investigated and the superiority of spot scanning method in both dose transfer and adaptation was investigated. According to the literature, there are some controversies regarding the distal dose of PSPT against PBS systems $(3,6$, 7); it might stem from the fact that the estimated physical/ biological dose in passive scattering proton therapy systems are dependent on the specific scattering hardware's that are implemented in the beam 
trajectory. In this regard, the performance of multiple scattering designs should be simulated and further compared to provide a global conclusion about the advantages and disadvantages of active scanning proton therapy technique against passive scattering mode. In the next step, it is better to do such a study in the tissue and in conditions such as the presence of implants to clarify the importance of therapeutic methods in dose delivery (and ability of PT mode to reduce the dose of secondary products).

\section{Abbreviations}

PT: proton therapy; PSPT:passive scattering proton therapy; PBS:pencil beam scanning; MC:Monte Carlo; SOBP:Spread-Out Bragg Peak; RBE:relative biological effectiveness; LET:Linear Energy Transfer; $\mathrm{BP}_{\text {W50 }}$ :Bragg Peak width 50; SAPT:Shanghai Advanced Proton Therapy; H\&N; head and neck; CTV:clinical target volume; PTV:planning target volume; MRE:mean relative error; IDD:Integrated Depth Dose; DVH:dose volume histogram; OARs:organ at risks.

\section{Declarations}

\section{Acknowledgment}

This work was supported by Sharif University of Technology and the Swiss National Science Foundation under grant SNRF 320030_176052.

\section{Authors' contributions}

All authors conceived the project. A Asadi and S A Hosseini conceived and designed the experiments, which were then performed by A Asadi. The data was analyzed and interpreted by A Asadi. All reagents, materials, analysis tools and data contributions were made by A Asadi and S A Hosseini. The paper was written by A Asadi and A Akhavanallaf, and all authors read and approved the final manuscript.

\section{Funding}

The present research work has not been founded by any organization.

\section{Availability of data and materials}

The data sets generated during the study are available from the corresponding author on reasonable request.

\section{Ethics Approval}


Not applicable.

\section{Consent for publication}

Not Applicable.

\section{Competing interests}

The authors declare that they have no competing interests.

\section{References}

1. Wilson RR. Radiological use of fast protons. Radiology. 1946;47(5):487-91.

2. ICRU P. Recording, and Reporting Proton-Beam Therapy International Commission on Radiation Units and Measurements Report 78. Bethesda, MD. 2007.

3. Michaelidesová A, Vachelová J, Klementová J, Urban T, Pachnerová Brabcová K, Kaczor S, et al. In Vitro Comparison of Passive and Active Clinical Proton Beams. Int J Mol Sci. 2020;21(16).

4. Kanai T, Kawachi K, Kumamoto Y, Ogawa H, Yamada T, Matsuzawa H, et al. Spot scanning system for proton radiotherapy. Medical physics. 1980;7(4):365-9.

5. Furukawa T, Inaniwa T, Sato S, Shirai T, Takei Y, Takeshita E, et al. Performance of the NIRS fast scanning system for heavy-ion radiotherapy. Medical physics. 2010;37(11):5672-82.

6. Nomura K, Iwata H, Toshito T, Omachi C, Nagayoshi J, Nakajima K, et al. Biological effects of passive scattering and spot scanning proton beams at the distal end of the spread-out Bragg peak in single cells and multicell spheroids. Int J Radiat Biol. 2021;97(5):695-703.

7. Gridley DS, Pecaut MJ, Mao XW, Wroe AJ, Luo-Owen X. Biological effects of passive versus active scanning proton beams on human lung epithelial cells. Technol Cancer Res Treat. 2015;14(1):81-98.

8. Shu H, Yin C, Zhang H, Liu M, Zhang M, Zhao L, et al. Scanned Proton Beam Performance and Calibration of the Shanghai Advanced Proton Therapy Facility. MethodsX. 2019;6:1933-43.

9. Paganetti H, Jiang H, Lee SY, Kooy H. Accurate Monte Carlo simulations for nozzle design, commissioning and quality assurance for a proton radiation therapy facility. Medical physics. 2004;31(7):2107-18.

10. Grevillot L, Bertrand D, Dessy F, Freud N, Sarrut D. A Monte Carlo pencil beam scanning model for proton treatment plan simulation using GATE/GEANT4. Physics in Medicine \& Biology. 2011;56(16):5203.

11. Gaizauskas R, Rodgers P, Cunningham H, Humphreys K, Robertson S. GATE User Guide. 1996.

12. Jan S, Benoit D, Becheva E, Carlier T, Cassol F, Descourt P, et al. GATE V6: a major enhancement of the GATE simulation platform enabling modelling of CT and radiotherapy. Physics in Medicine \& Biology. 2011;56(4):881. 
13. Mynampati DK, Yaparpalvi R, Hong L, Kuo HC, Mah D. Application of AAPM TG 119 to volumetric arc therapy (VMAT). Journal of applied clinical medical physics. 2012;13(5):108-16.

14. Bourhaleb F, Marchetto F, Attili A, Pittà G, Cirio R, Donetti M, et al. A treatment planning code for inverse planning and $3 \mathrm{D}$ optimization in hadrontherapy. Computers in biology and medicine. 2008;38(9):990-9.

15. Sánchez-Parcerisa D, López-Aguirre M, Dolcet Llerena A, Udías JM. MultiRBE: Treatment planning for protons with selective radiobiological effectiveness. Medical physics. 2019;46(9):4276-84.

16. Ezzell GA, Burmeister JW, Dogan N, LoSasso TJ, Mechalakos JG, Mihailidis D, et al. IMRT commissioning: multiple institution planning and dosimetry comparisons, a report from AAPM Task Group 119. Medical physics. 2009;36(11):5359-73.

17. de Oncología Radioterápica SE. Manual Práctico de Oncología Radioterápica. Madrid, Grupo Editorial abbvie. 2013;201.

18. Mendenhall NP, Li Z, Hoppe BS, Marcus Jr RB, Mendenhall WM, Nichols RC, et al. Early outcomes from three prospective trials of image-guided proton therapy for prostate cancer. International Journal of Radiation Oncology* Biology* Physics. 2012;82(1):213-21.

\section{Figures}

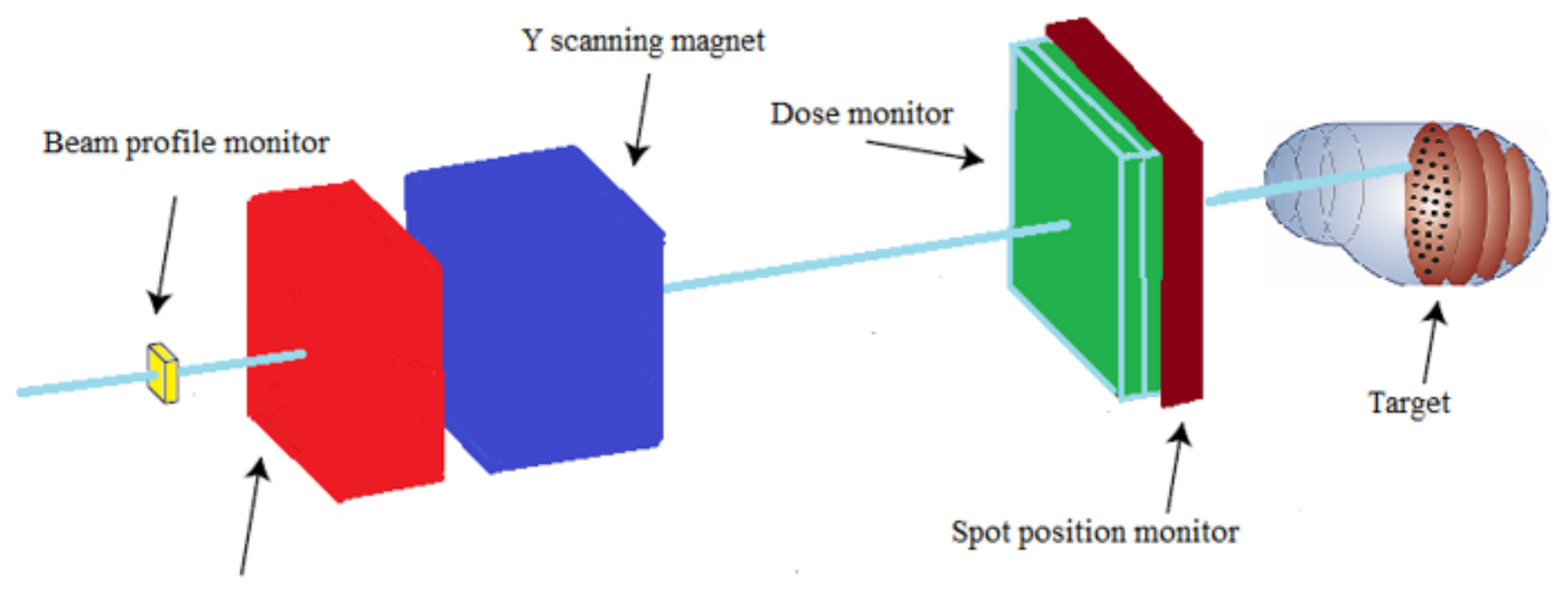

$\mathrm{X}$ scanning magnet

\section{Figure 1}

A schematic view of SAPT nozzle. 


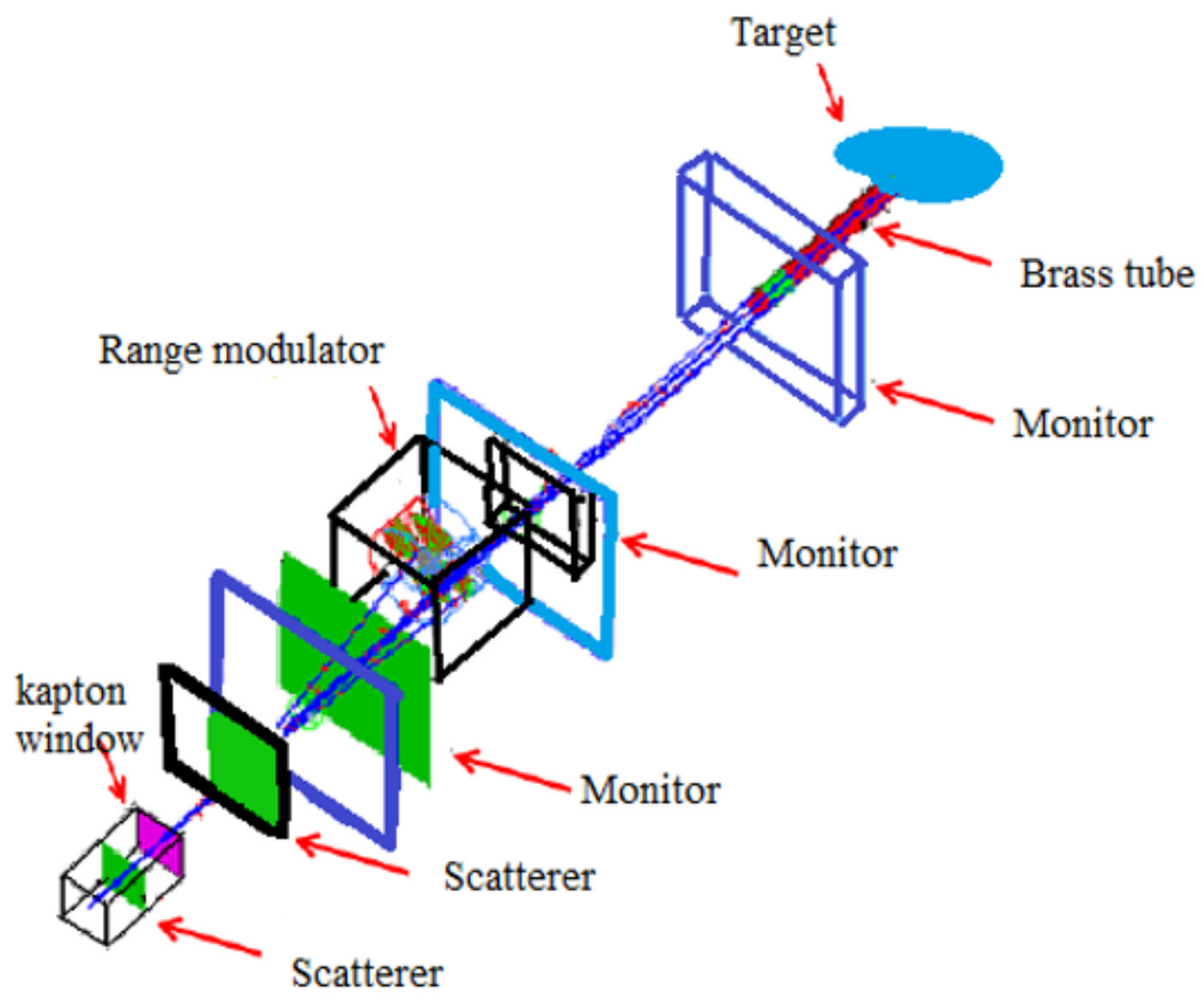

Figure 2

A schematic view of passive scattering system including scatterer, i.e. range modulator, collimator and compensator, along with monitoring components.
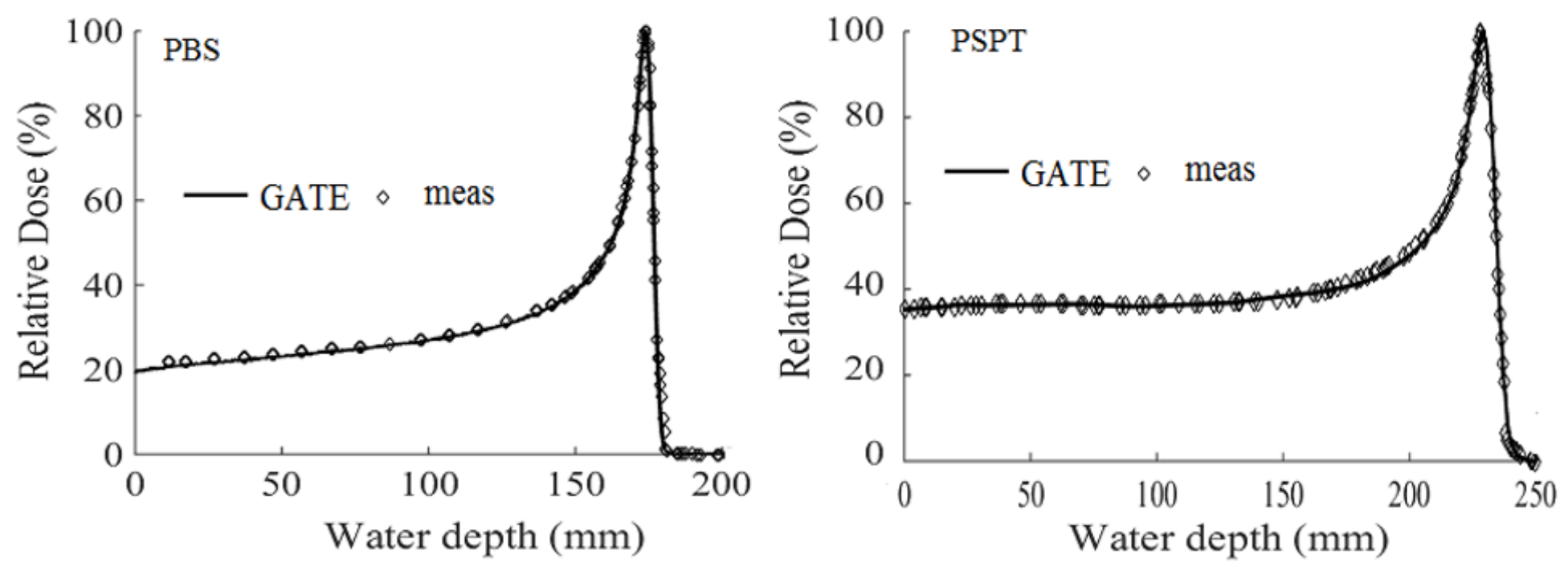

Figure 3 
Comparison between the simulated and experimental data for IDD parameters, in the active scan-based method (left), and scatter-based method (right).
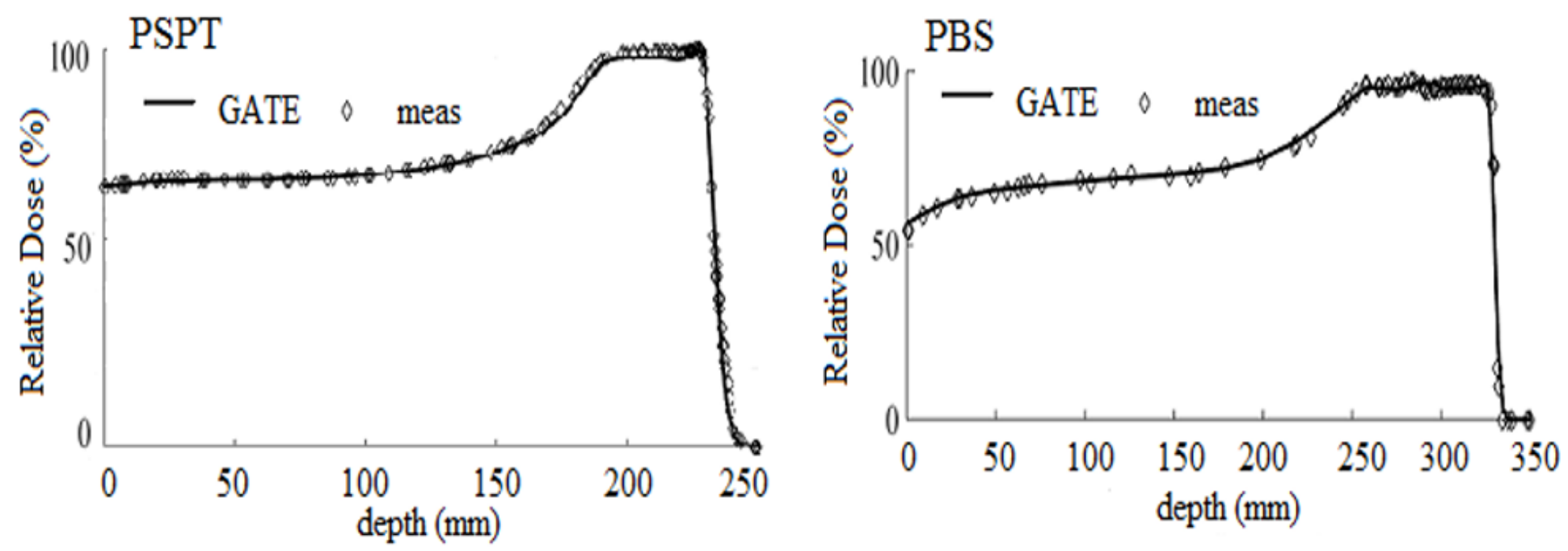

Figure 4

Comparison between the simulated and experimental data for SOBP plan, in the scatter-based method (left), and active scan-based method (right).

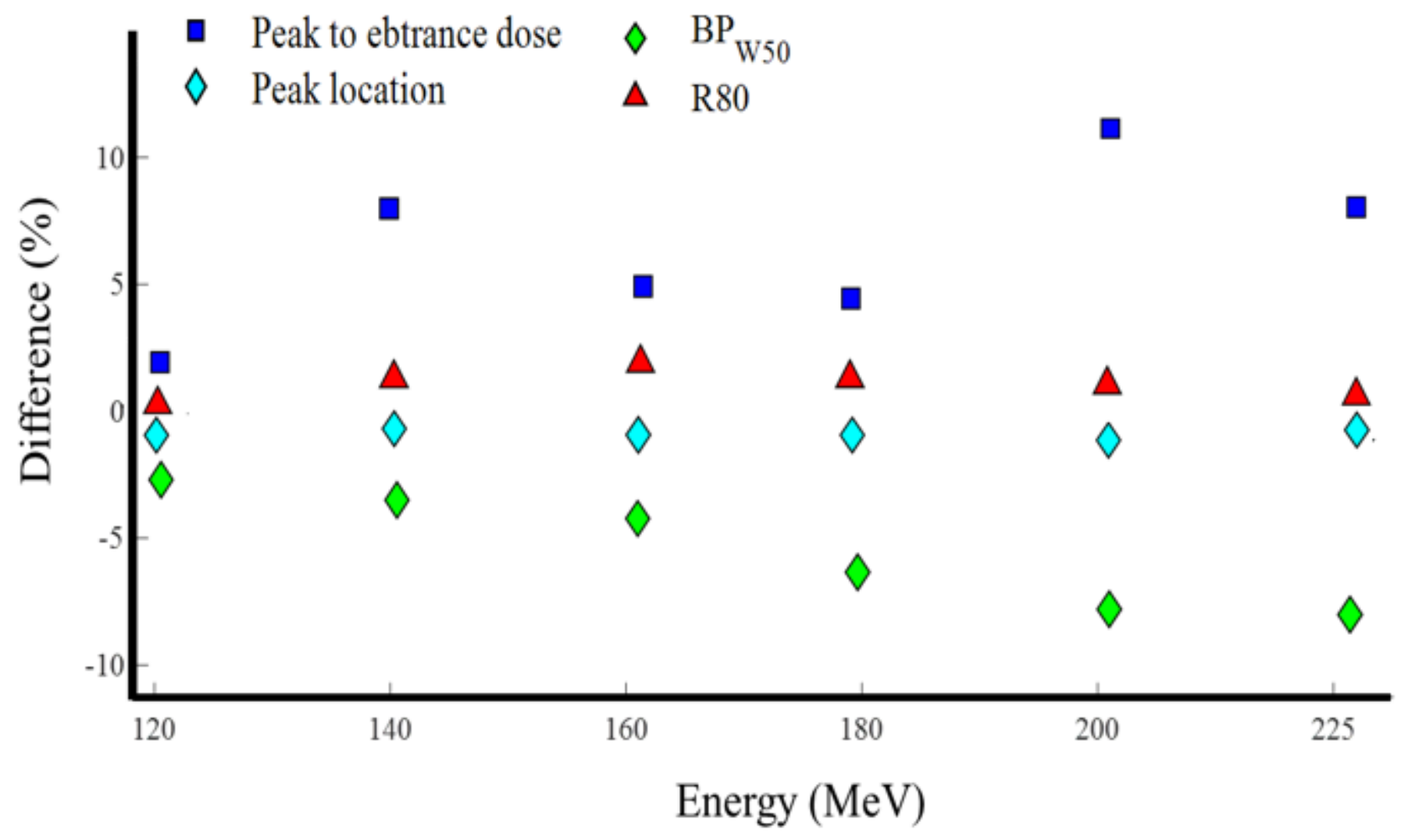

Figure 5

Please see the Manuscript file for the complete figure caption. 

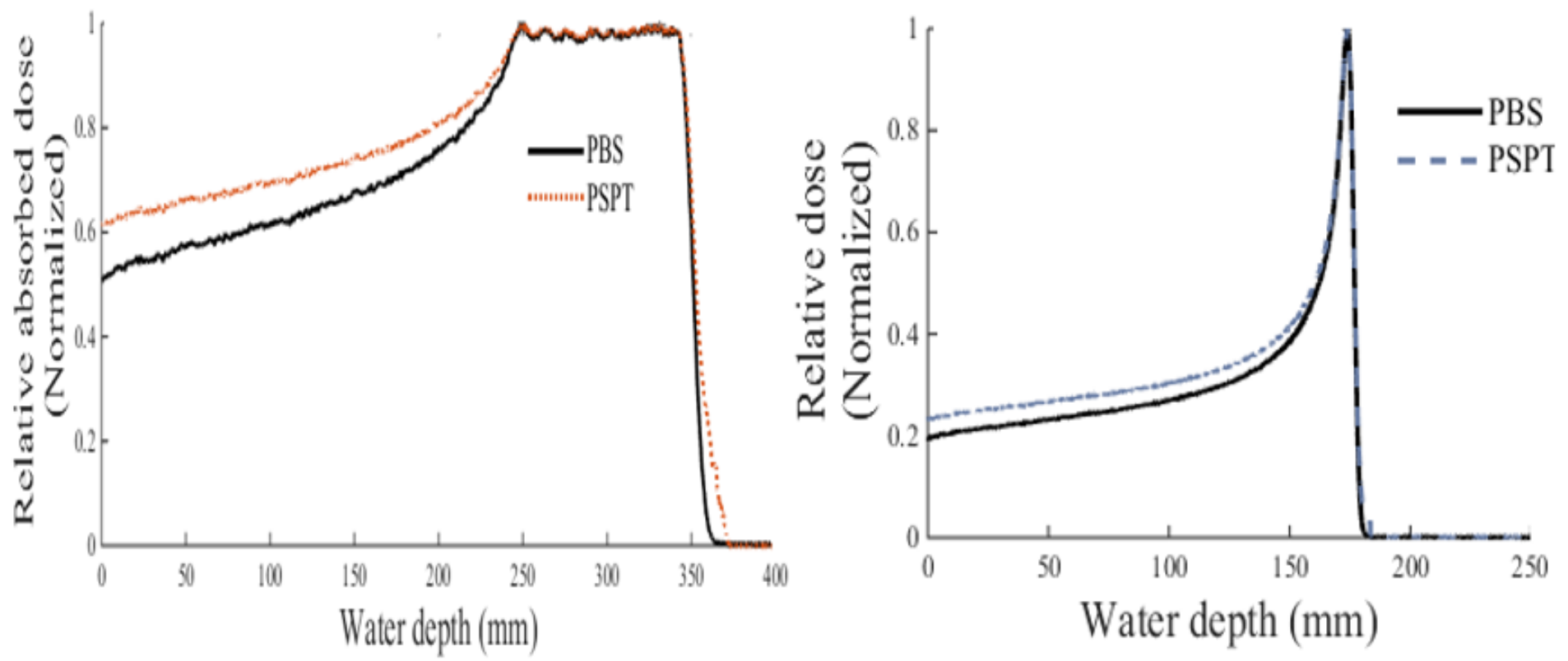

Figure 6

IDD (right) and SOBP (left) diagram obtained from simulated PBS and PSPT systems in proton energy of $179.9 \mathrm{MeV}$.

PBS
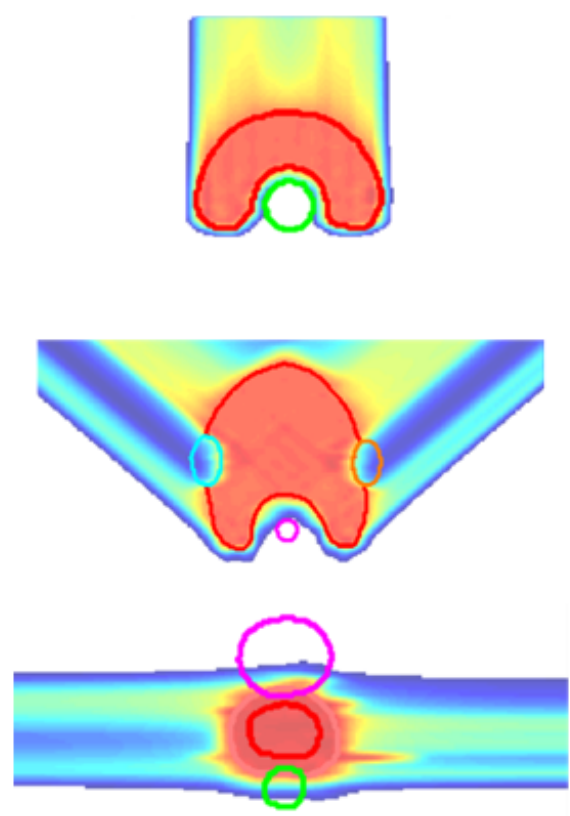

PSPT
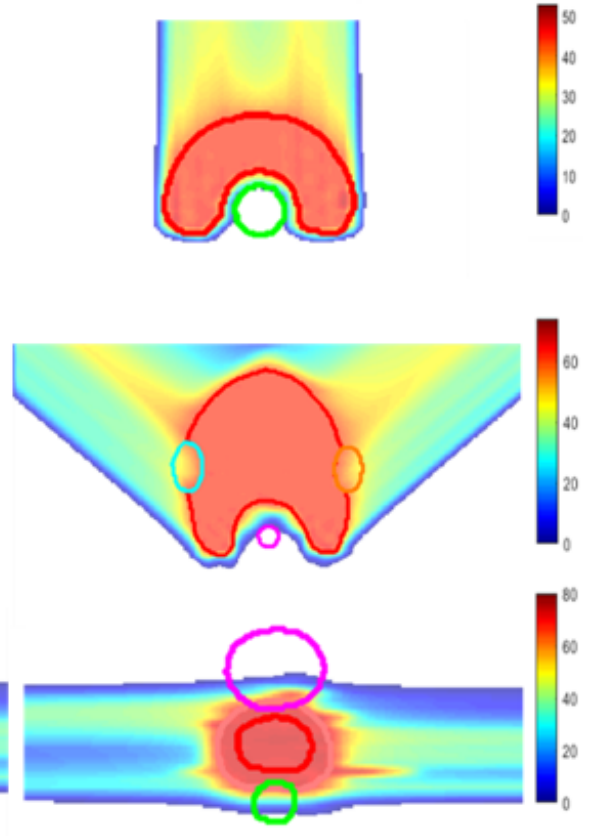

Difference
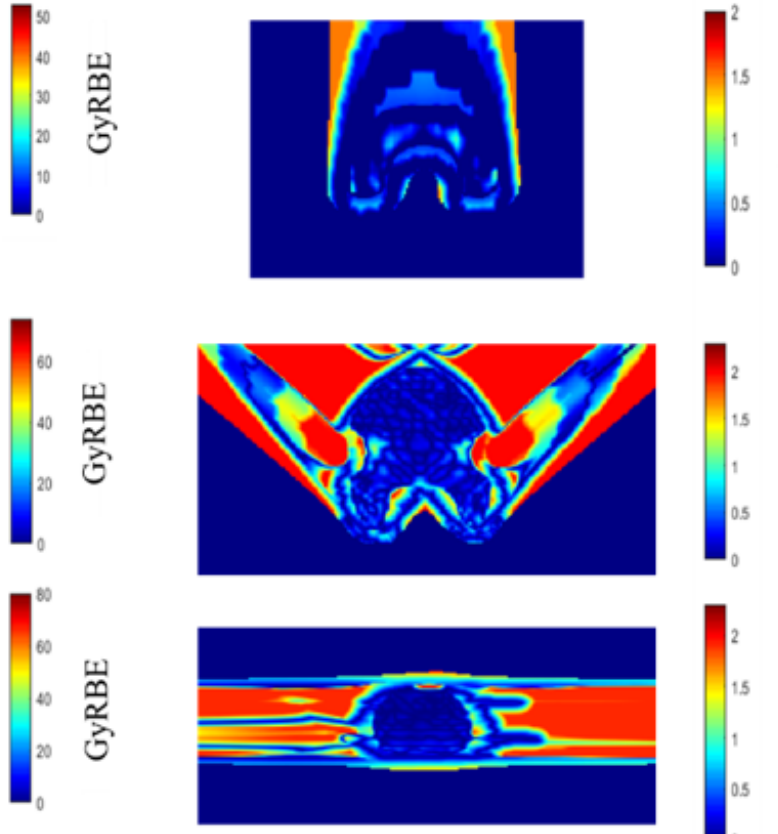

Figure 7

3D dose distribution and comparison for PBS and PSPT plans. 

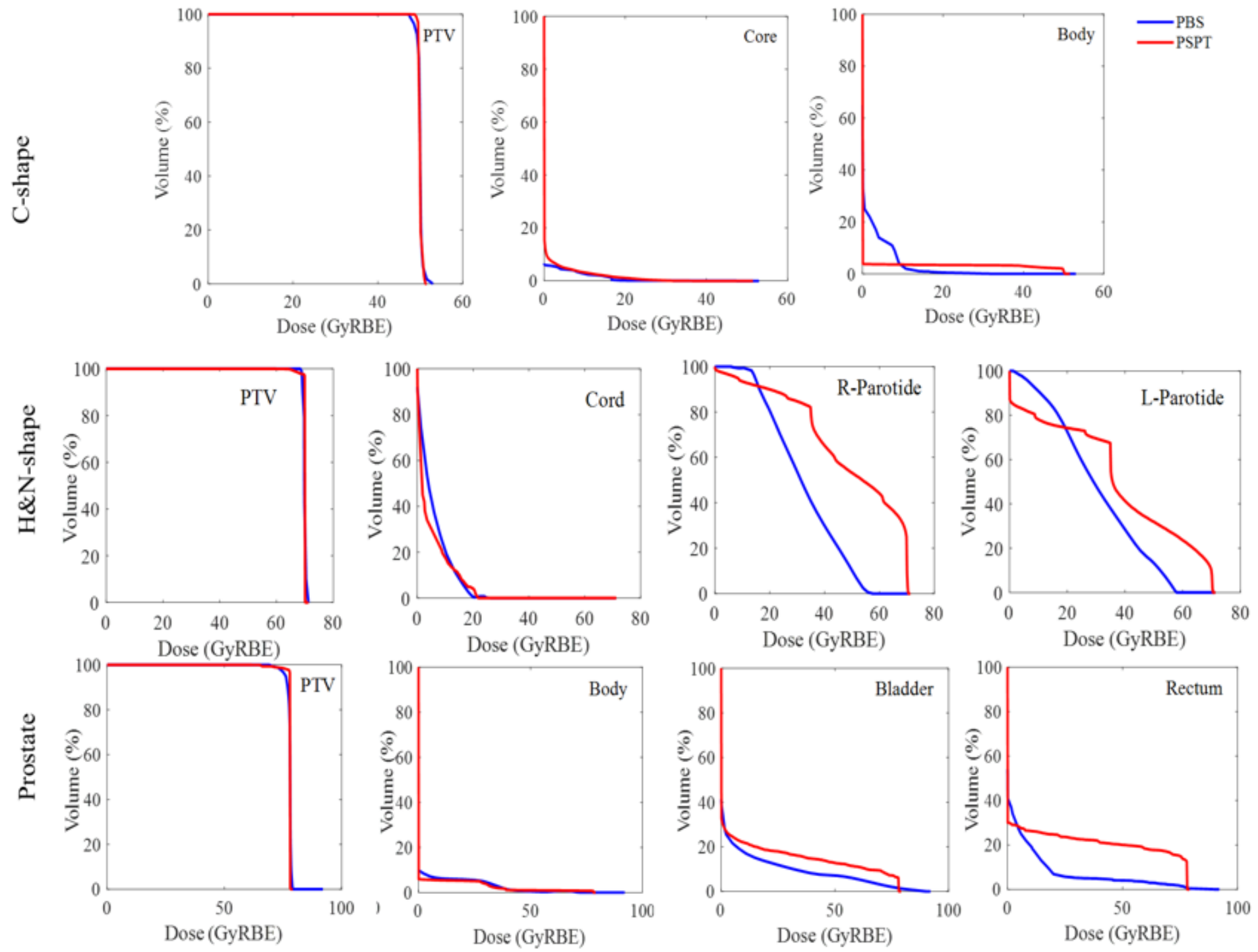

\section{Figure 8}

DVH analysis for PBS (blue line) and PSPT (red line) plans. 